1 Universidade Federal da Bahia (UFBA), Instituto Multidisciplinar em

Saúde, Programa de

Pós-Graduação em Saúde

Coletiva - Vitória da Conquista (BA), Brasil.

Orcid: https://orcid. org/0000-0001-8243-

5662

nilia.prado@ufba.com

2 Universidade Federal da Bahia (UFBA), Instituto

Multidisciplinar em

Saúde, Programa de

Pós-Graduação em Saúde

Coletiva - Vitória da

Conquista (BA), Brasil.

Orcid: https://orcid.

org/0000-0001-9718-

1562

maiaufba@ufba.br

\section{Promoção da saúde na Atenção Primária à Saúde: sistematização de desafios e estratégias intersetoriais}

\author{
Health promotion in Primary Health Care: systematization of \\ challenges and intersectoral strategies
}

Nilia Maria de Brito Lima Prado', Adriano Maia dos Santos²

DOI: $10.1590 / 0103-110420185126$

RESUMO Foi realizada revisão da literatura com o intuito de sistematizar o conhecimento produzido no campo da promoção da saúde com ênfase nos desafios e estratégias para implementação da intersetorialidade. As bases de dados consultadas foram Lilacs, SciELO, Web of Science e Science Direct; e identificadas publicações referentes ao período compreendido entre 2006 e 2017. O corpus contou com 28 artigos, sendo analisados os aspectos relacionados com os resultados dos estudos. Verificou-se que eles têm destacado a discussão de fragilidades contextuais relacionadas com aspectos gerenciais e operacionais das intervenções para promoção da saúde, assim como com estratégias adotadas para fomentar a implementação e gestão de ações intersetoriais. Conclui-se que são necessários investimentos em estudos de caso que permitam analisar intervenções concretas direcionadas à promoção da saúde, agregar novas explicações e estabelecer coerência com a complexidade dos distintos contextos no âmbito da Atenção Primária à Saúde.

PALAVRAS-CHAVE Atenção Primária à Saúde. Promoção da saúde. Colaboração intersetorial. Revisão.

ABSTRACT A review of the literature was carried out in order to systematize the knowledge produced in the field of health promotion with an emphasis on the challenges and strategies for the implementation of intersectoriality. The databases consulted were Lilacs, SciELO, Web of Science and Science Direct and identified publications referring to the period between 2006 and 2017. The corpus had 28 articles, analyzing the aspects related to the methodology and results of the studies. It was verified that the studies have highlighted the discussion of contextual weaknesses related to managerial and operational aspects of health promotion interventions. It is concluded that investments are necessary in case studies that allow analyzing concrete interventions aimed at health promotion, adding new explanations and establishing coherence with the complexity of the different contexts in Primary Health Care.

KEYWORDS Primary Health Care. Health promotion. Intersectoral collaboration. Review. 


\section{Introdução}

O desenvolvimento de ações para a promoção da saúde no âmbito da Atenção Primária à Saúde (APS) desponta como importante resposta à reestruturação do modelo de atenção à saúde e operacionalização de ações que priorizam uma abordagem fundamentada nos determinantes sociais da saúde $\mathbf{1}^{\mathbf{1}} \mathbf{2}$. Nessa perspectiva, a promoção da saúde seria a estratégia preferencial para viabilizar a articulação de conhecimentos interdisciplinares no cuidado individual e coletivo, bem como a APS seria o lócus privilegiado para mobilização comunitária, capaz de propiciar mudanças de cultura organizacional, ampliação do escopo de ações e a reorganização dos sistemas locais de saúde 3 .

No Brasil, as ações para promoção da saúde emergiram como desdobramento do esforço para a qualificação do Sistema Único de Saúde (SUS) e do debate sobre as relações entre iniquidades sociais, democracia, desenvolvimento humano e saúde no campo da saúde coletiva ${ }^{4}$. Nesse sentido, a Estratégia Saúde da Família (ESF) emergiu na dinâmica do território como um espaço permanente de construção e reconstrução de processos relacionais que revela a pluralidade e, sobretudo, a potência dos atores para identificar necessidades e fomentar as ações de promoção da saúde ${ }^{5}$.

Embora se ressalte a contribuição da ESF nesse particular, as ações desenvolvidas na APS têm apresentado limites gerenciais e operacionais em relação ao enfrentamento do conjunto de determinantes da saúde e ao enfoque sociopolítico da promoção da saúde ${ }^{6}$. Poucos estudos no Brasil problematizaram ou avaliaram a natureza e efetividade das ações de promoção da saúde desenvolvidas no âmbito da APS7.

Da mesma forma, as análises sobre experiências de intersetorialidade implementadas em contextos diversificados ainda são raras na literatura acadêmica, sendo premente a identificação e análise do conhecimento científico produzido sobre as condições que influenciam uma gestão eficaz de ações intersetoriais ${ }^{8-10}$. Portanto, fazem-se necessários o debate e a problematização acerca dos caminhos, das implicações, dos limites e perspectivas que se colocam para a consolidação das práticas de promoção da saúde no âmbito da APS, cenário imprescindível para a consolidação do SUS, tão ameaçado pela conjuntura atual em nosso país.

Diante da complexidade da discussão, este artigo busca sistematizar o conhecimento produzido no campo da promoção da saúde com ênfase nos desafios e estratégias para a implementação da intersetorialidade.

\section{Metodologia}

Trata-se de uma revisão integrativa da literatura sobre os desafios e estratégias para a implementação de ações intersetoriais para promoção da saúde.

Realizou-se busca em bases eletrônicas de publicações científicas, sendo identificadas as seguintes: Scientific Electronic Library Online (SciELO), Literatura Latino-Americana e do Caribe em Ciências da Saúde (Lilacs), Web of Science e Science Direct. Como período de busca, utilizou-se de março de 2006 a dezembro de 2017. Optou-se por tal período, pois, em março de 2006, foi publicada a primeira versão da Política Nacional de Promoção da Saúde (PNPS). A coleta de dados ocorreu em dezembro de 2017.

Os unitermos ou descritores foram previamente identificados nos Descritores em Ciências da Saúde (DeCS) e Medical Subject Heading (MeSH), sendo: 'Primary Health Care', 'Health promotion', 'Intersectoral action', 'Health Management'. Em seguida, foi feita uma combinação por meio do conectores boleanos: Health Promotion AND Primary Health Care AND Health Management; Intersectoral Action AND Primary Health Care AND Health Management; Health Promotion AND Health Management AND Intersectoral action; Health Promotion AND Intersectoral Action AND Primary Health Care AND Health Management. Foram eleitos artigos nos idiomas inglês, espanhol e português. A 
busca de documentos se deu por meio da ferramenta 'busca integrada', sendo selecionados artigos que estavam indexados na íntegra.

Foram utilizados como critério de inclusão para seleção dos artigos: estudos que abordassem ações intersetoriais para promoção da saúde ou intervenções intersetoriais problemas ou condições relacionadas com a saúde, quais sejam: obesidade, tabagismo e álcool, prevenção de cuidados dentais, doenças crônicas, segurança alimentar e nutricional, incluindo a alimentação escolar e as ações relacionadas ao Programa Saúde na Escola (PSE), promoção da atividade física e práticas corporais e educação permanente em saúde; estudos empíricos e/ou de revisão e/ou conceitual na área de ação e gestão intersetorial para promoção da saúde, publicados em revistas científicas, não abrangendo, portanto, possíveis intervenções derivadas de projetos de pesquisa realizados em território nacional e internacional; publicados nas bases de dados no período definido e na língua portuguesa, inglesa ou espanhola.

Já como critérios de exclusão, utilizaram-se: os estudos que não investigaram ações intersetoriais para promoção da saúde; as publicações de editoriais e anais de congressos científicos; e os estudos que abordaram outras ações específicas que não referiam ações intersetoriais.

Para viabilizar a definição dos artigos selecionados, uma base de dados única foi construída em planilha Excel $^{\circledR}$ que reuniu um total de 839 artigos, explorados inicialmente por meio da leitura de títulos e resumos, sendo identificados 269 artigos duplicados, os quais foram excluídos, ficando, assim, a análise crítica de 570 artigos completos. Após leitura dos artigos na íntegra, foram excluídos 355 artigos que não discutiam ações intersetoriais, apenas referiam-se a ações assistenciais vinculadas a equipes de saúde, restando 215 artigos que foram lidos e analisados quanto à identificação dos assuntos considerados centrais (desafios e estratégias para fomentar ações intersetoriais para a promoção da saúde); e excluídos 187 que não apresentavam a discussão de tais assuntos e apenas descreviam ações relacionadas com programas assistenciais. Ao final, foram incluídos neste estudo 28 artigos.

Cabe salientar que a seleção e inclusão dos estudos nesta revisão foi realizada em consenso com os dois autores que avaliaram e selecionaram os artigos que continham o desfecho de interesse.

A qualidade das publicações incluídas na revisão foi aferida por meio da aplicação do Consolidated Criteria for Reporting Qualitative Studies (Coreq) ${ }^{11}$, com alcance de 24 itens dentro dos 32 solicitados (apenas os itens referentes a equipe de pesquisa não se aplicaram a esse tipo de revisão).

$\mathrm{Na}$ análise dos artigos, dois eixos temáticos foram criados como categorias emergentes: a. Condições contextuais restritivas à implementação e gestão de ações intersetoriais e, b. Estratégias adotadas para fomentar a implementação e gestão de ações para promoção da saúde. Cada artigo foi analisado segundo esses eixos, o ano de publicação, o país de estudo, metodologia adotada e população do estudo sistematizados no quadro 1.

\section{Resultados e discussão}

A distribuição temporal das 28 publicações analisadas foi a seguinte: 3 artigos de 2009; 3 de 2010; 1 de 2011; 1 de 2012; 13 de 2014; 3 de 2015; 3 de 2016 e 1 de 2018. No que se refere aos locais do estudo: 15 foram do Brasil; 3, do Canadá; 3, da Austrália; 2, da Noruega; 1 foi dos Estados Unidos; 1, da Alemanha; 1, da França; 1, da Holanda e 1, da Suíça em cooperação com o Brasil. Sobre o desenho: 19 foram estudos com metodologia qualitativa (estudo de caso=16; estudos avaliativos=3); 5 foram revisão de literatura; 1 foi pesquisa documental/revisão documental; 1 foi estudo transversal com dados secundários e 2 estudos adotaram ambas as metodologias qualitativa e quantitativa (quadro 1). 
Quadro 1. Descrição dos artigos incluídos na revisão de literatura, segundo autor, ano, país de origem, metodologia, população estudada e condições contextuais e estratégias para implementação e gestão de ações intersetoriais para promoção da saúde

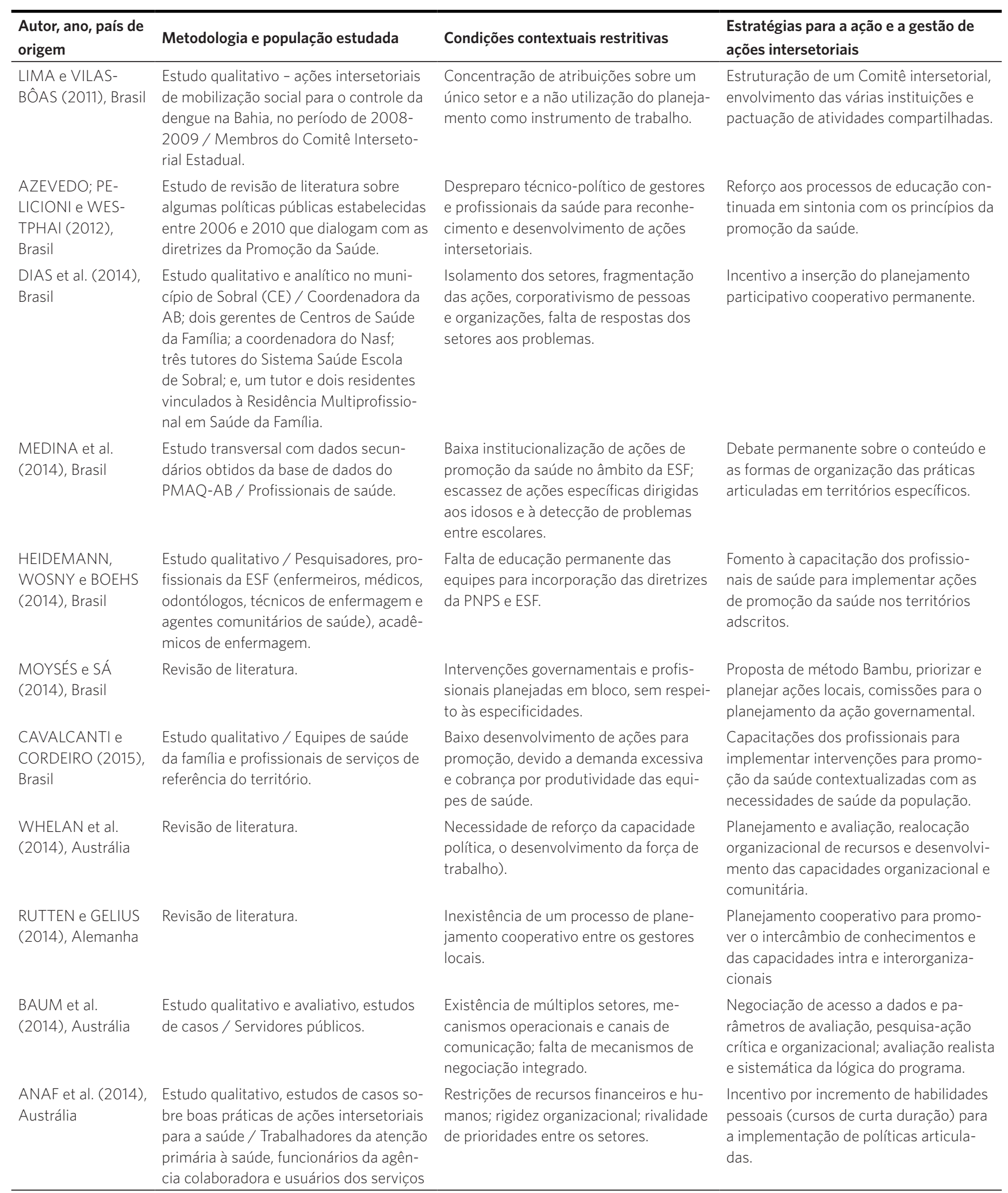




\begin{tabular}{|c|c|c|c|}
\hline \multicolumn{4}{|l|}{ Quadro 1. (cont.) } \\
\hline $\begin{array}{l}\text { LITTLEJOHNS e } \\
\text { SMITH (2014), } \\
\text { Canadá }\end{array}$ & $\begin{array}{l}\text { Estudo qualitativo / Gestores munici- } \\
\text { pais. }\end{array}$ & $\begin{array}{l}\text { Dificuldade em estabelecer ações coor- } \\
\text { denadas e com maior durabilidade em } \\
\text { longo prazo. }\end{array}$ & $\begin{array}{l}\text { Fortalecimento da ação comunitária, } \\
\text { desenvolvimento de competências } \\
\text { pessoais e reorientação dos serviços de } \\
\text { saúde; conectividade e de coesão. }\end{array}$ \\
\hline $\begin{array}{l}\text { STORM et al. } \\
\text { (2014), Holanda }\end{array}$ & $\begin{array}{l}\text { Estudo qualitativo e avaliativo / Gestores } \\
\text { municipais e profissionais de saúde. }\end{array}$ & $\begin{array}{l}\text { Falta de conhecimento para avaliar polí- } \\
\text { ticas intersetoriais. }\end{array}$ & $\begin{array}{l}\text { Desenvolvimento de software para ge- } \\
\text { renciamento de projetos articulados. }\end{array}$ \\
\hline $\begin{array}{l}\text { GREER e LILLVIS } \\
\text { (2014), Estados } \\
\text { Unidos }\end{array}$ & $\begin{array}{l}\text { Estudo qualitativo, estudo de caso / } \\
\text { Formuladores e gestores de políticas } \\
\text { governança intersetorial. }\end{array}$ & $\begin{array}{l}\text { Dificuldade em estabelecer ações coor- } \\
\text { denadas com sustentabilidade em longo } \\
\text { prazo. }\end{array}$ & $\begin{array}{l}\text { Mudanças burocráticas, como a introdu- } \\
\text { ção da Avaliação do Impacto na Saúde } \\
\text { ou reorganização; e métodos indiretos } \\
\text { como publicação de dados e apoio de } \\
\text { grupos externos. }\end{array}$ \\
\hline $\begin{array}{l}\text { ZICKER et al. } \\
\text { (2015), Brasil e } \\
\text { Suíça }\end{array}$ & $\begin{array}{l}\text { Estudo qualitativo em plataforma virtual } \\
\text { / Coordenadores de iniciativas globais } \\
\text { de saúde. }\end{array}$ & $\begin{array}{l}\text { Conhecimento insuficiente para o pla- } \\
\text { nejamento de ações integradas entre os } \\
\text { atores e setores. }\end{array}$ & $\begin{array}{l}\text { Otimização de plataformas de gestão } \\
\text { de conhecimento; translação de conhe- } \\
\text { cimento. }\end{array}$ \\
\hline $\begin{array}{l}\text { TROMPETTE et al. } \\
\text { (2014), França }\end{array}$ & $\begin{array}{l}\text { Estudo qualitativo, estudo de caso / } \\
\text { Agentes de promoção da saúde envolvi- } \\
\text { dos em um programa regional. }\end{array}$ & Existência de intervenções, antagônicas. & $\begin{array}{l}\text { Inclusão de um plano de ação integrado } \\
\text { entre os setores. }\end{array}$ \\
\hline $\begin{array}{l}\text { TESS e AITH } \\
\text { (2014), Brasil }\end{array}$ & $\begin{array}{l}\text { Estudo qualitativo e quantitativo, estudo } \\
\text { de caso / Decisores políticos e gestores } \\
\text { municipais. }\end{array}$ & $\begin{array}{l}\text { Estruturas organizacionais e institucio- } \\
\text { nais fragmentadas, sem coordenação de } \\
\text { informações. }\end{array}$ & $\begin{array}{l}\text { Estabelecimento de coordenação com- } \\
\text { partilhada das políticas intersetoriais. }\end{array}$ \\
\hline $\begin{array}{l}\text { HENDRIKS et al. } \\
\text { (2015), Holanda }\end{array}$ & $\begin{array}{l}\text { Estudo qualitativo, estudo de caso / } \\
\text { Funcionários responsáveis por diferentes } \\
\text { setores de políticas em dois municípios } \\
\text { de pequeno porte. }\end{array}$ & $\begin{array}{l}\text { Existência de estruturas organizacionais } \\
\text { fragmentadas. }\end{array}$ & $\begin{array}{l}\text { Reorganização de estrutura organizacio- } \\
\text { nal e treinamento de funcionários pelos } \\
\text { gerentes. }\end{array}$ \\
\hline $\begin{array}{l}\text { WEISS, LILLEFJELL } \\
\text { e MAGNUS (2016), } \\
\text { Noruega. }\end{array}$ & Revisão de literatura. & $\begin{array}{l}\text { Estruturas organizacionais e institucio- } \\
\text { nais fragmentadas. }\end{array}$ & $\begin{array}{l}\text { Fomento a formação de rede de gestores } \\
\text { capacitados para o monitoramento e } \\
\text { avaliação de ações intersetoriais. }\end{array}$ \\
\hline $\begin{array}{l}\text { KOTHARI et al. } \\
\text { (2016), Canadá }\end{array}$ & $\begin{array}{l}\text { Estudo qualitativo, estudo de múltiplos } \\
\text { casos/ Profissionais de saúde de quatro } \\
\text { redes de saúde pública. }\end{array}$ & $\begin{array}{l}\text { Baixa competência dos gestores e profis- } \\
\text { sionais locais. }\end{array}$ & $\begin{array}{l}\text { Desenvolvimento de planos de ação } \\
\text { intersetorial local. }\end{array}$ \\
\hline $\begin{array}{l}\text { GUGLIELMIN et al. } \\
\text { (2018), Canadá }\end{array}$ & Revisão de literatura. & $\begin{array}{l}\text { Restrições de recursos financeiros e } \\
\text { humanos; rigidez organizacional; falta de } \\
\text { financiamento e de liderança local. }\end{array}$ & $\begin{array}{l}\text { Fomento à formação de gestores para o } \\
\text { planejamento, monitoramento e avalia- } \\
\text { ção de ações intersetoriais. }\end{array}$ \\
\hline
\end{tabular}

Fonte: Elaboração própria.

Os principais atores sociais envolvidos na implementação de intervenções intersetoriais referidos pelos artigos eram os gestores estaduais e municipais e os profissionais de saúde atuantes em equipes de saúde no âmbito da APS ${ }^{12-15}$. Por outro lado, apesar da importância do fortalecimento da autonomia do coletivo para a tomada das decisões, grande parte dos estudos evidenciou uma participação comunitária pouco expressiva ${ }^{16-19}$.
As intervenções para promoção da saúde descritas abrangeram experiências locorregionais direcionadas à redução de iniquidades e ampliação do acesso equânime a bens e serviços de saúde de grupos populacionais em distintos contextos, nos quais as fragilidades do contexto sociopolítico, administrativo e técnico poderiam estar restringindo as mudanças sociais mais abrangentes e em longo prazo. 


\section{Condições contextuais à im- plementação e gestão de ações intersetoriais}

Neste subtópico, serão apresentadas as condições contextuais consideradas restritivas à implementação e gestão de ações intersetoriais. De modo geral, os contextos de implementação das intervenções analisadas discorreram especialmente sobre três questões transversais.

Primeiro, a inviabilidade do contexto organizacional e sociopolítico para o desenvolvimento de ações intersetoriais (desenvolvimento de ações intersetoriais governamentais planejadas sem respeito às especificidades locorregionais e pela inviabilidade política; falta de processo de planejamento e intercâmbio de conhecimentos entre os decisores políticos vinculados à gestão da intervenção, processo de implementação não linear; ausência de mecanismos de negociação).

Segundo, em relação ao despreparo político e técnico dos gestores para assumir e coordenar o planejamento, monitoramento e avaliação de intervenções (incipiente capacitação dos atores sociais para o desenvolvimento de intervenções intersetoriais; ausência de financiamento exclusivo para custear as ações articuladas entre os setores; desconhecimento dos gestores sobre mecanismos para mensuração dos resultados alcançados).

Terceiro, a incipiente capacidade dos profissionais de saúde da APS para a construção de agendas integradas e para a formação de novas redes sociais capazes de operar em territórios específicos para a execução de ações integradas para a promoção da saúde coerentes com as necessidades locorregionais, especialmente pela existência de rigidez organizacional e rivalidade de prioridades entre os setores.

A análise das experiências, a partir dessas questões, permitiu evidenciar alguns fatores restritivos relacionados com o contexto sociopolítico, com a gestão municipal de saúde e com a inserção da equipe de saúde em território predefinido.

\section{INVIABILIDADE DO CONTEXTO ORGANIZACIONAL E SOCIOPOLÍTICO PARA O DESENVOLVIMENTO DE AC̣ÕES INTERSETORIAIS}

Os principais desafios foram as estruturas hierarquizadas identificadas na maioria das experiências municipais, tendo em vista que alguns estudos identificaram a realização de ações previstas pelos programas sujeitas a interesses em jogo e aos processos que privilegiavam determinadas políticas setoriais que permeavam as instituições.

Foram constatadas fronteiras reais à integração da APS na rede assistencial, indicando a baixa governabilidade de alguns municípios sobre parte dos serviços especializados, sob gestão estadual; e a insuficiente Programação Pactuada e Integrada para reduzir a fragmentação entre as redes estadual e municipal, por não haver garantias de que as cotas programadas de procedimentos fossem distribuídas entre as unidades de saúde ${ }^{10}$.

Os fatores desfavoráveis à consolidação do novo modelo tecnoassistencial em saúde foram ressaltados também em um estudo realizado com profissionais atuantes na APS de Belo Horizonte e Contagem ${ }^{12}$. As reflexões dos entrevistados indicaram que a oferta de serviços é estruturada em torno de práticas assistenciais curativas que, muitas vezes, não abrangiam as necessidades de saúde dos usuários adscritos. Para os autores, o critério de produtividade exigido para alcance do cumprimento das metas e indicadores na obtenção de resultados mostrou interferência no processo de trabalho das equipes considerando a lógica da ESF. Esse modelo demanda em ações pouco integradas ao processo de trabalho e diminuição do grau de autonomia profissional.

Outros fatores restritivos mais específicos foram apontados pelo estudo realizado por Magalhães e Bodstein ${ }^{15}$, que destacou a falta de comunicação e as 
articulações descontínuas entre os atores e setores como os aspectos mais frágeis, pois constatavam um planejamento de ações incoerentes com as necessidades de saúde da população, questão referida como passível de contribuir para a sobreposição de ações no território, assim como por limitar o alcance das intervenções. Por outro lado, apontaram que as iniciativas intersetoriais foram mais abrangentes quando definidas como política integrada do governo municipal para a construção de interfaces e cooperação entre os diversos setores.

Silva e Rodrigues ${ }^{16}$ versam que a intersetorialidade ainda se constitui como um objetivo a ser alcançado. As autoras reconhecem que, em um território, há várias possibilidades de articulação em rede, contudo, para que o serviço de saúde local possa, de fato, estabelecer parcerias intersetoriais, é imprescindível ir além da negociação de tarefas e da transferência de responsabilidades.

Um estudo realizado por Medina et al. ${ }^{17}$, que descreveu as ações de promoção da saúde e prevenção de doenças crônicas realizadas pelas equipes de saúde que aderiram ao Programa Nacional de Melhoria do Acesso e da Qualidade da Atenção Básica (PMAQ-AB) no Brasil, demonstrou a incipiência da realização das ações analisadas, especialmente aquelas dirigidas para idosos e para a detecção de problemas entre escolares, além de pouca comprovação das ações realizadas, traduzindo a baixa institucionalização dessas ações no âmbito da ESF.

O desenvolvimento de ações intersetoriais governamentais planejadas sem respeito às especificidades locorregionais - e, especialmente, a falta de autonomia dos entes federados municipais para a operacionalização das ações derivadas das diretrizes expressas na PNPS, particularmente, a versão publicada no ano de 2006, do mesmo modo - foi amplamente referido pelos artigos ${ }^{10-17}$. Nesse sentido, a falta de autonomia, segundo tais estudos, impedia a implementação e gestão de ações articuladas ao limitar a coordenação política e as atribuições dos governos locais e por condicionar os sistemas locais a uma forte dependência de recursos financeiros dos governos nos âmbitos estadual e nacional18-22.

A conformação das ações para promoção da saúde na ESF a partir de um território na cidade do Recife evidenciou uma prática intersetorial deficitária. Os resultados apontaram o diálogo entre os setores como possibilidade para potencializar as ações articuladas $^{21}$. Os autores indicaram que o desafio é instituir a cultura da articulação permanente entre setores distintos e unir esforços para transformar o que, hoje, é reconhecido como importante e útil, para além dos discursos de suas vantagens, partindo para um planejamento conjunto e sistematizado de ações condicionadas às necessidades identificadas localmente.

\section{DESPREPARO POLÍTICO E TÉCNICO DOS GESTORES E PROFISSIONAIS PARA ASSUMIR E COORDENAR O PLANEJAMENTO, MONITORAMENTO E AVALIAÇÃO DE INTERVENÇÕES INTERSETORIAIS}

Um estudo desenvolvido por Silva e Tavares ${ }^{23}$ reforçou a dificuldade da gestão local para atender e incluir os critérios estabelecidos por portarias regulamentadoras, que nem sempre são prioritários para as comunidades envolvidas e acabam por constituírem ações verticalizadas sem um planejamento conjunto entre os entes federados, o que pode contribuir para a dependência contínua aos demais níveis de gestão federativa. As limitações da gestão local, segundo os autores, referiam-se à transferência apenas de autoridade administrativa, sem evidenciar uma autonomia local.

Outro ponto crítico considerado por alguns estudos diz respeito às dificuldades para o desenvolvimento de um trabalho articulado em territórios adscritos à ESF. Nesse ínterim, a incipiente capacidade técnica dos profissionais de saúde para 
estabelecer alianças estratégicas capazes de garantir a incorporação de diretrizes expressas nas políticas e procedimentos organizacionais orientados pelo estado para implementação em territórios vinculados à APS foi mencionada ${ }^{23-26}$.

A disputa por centralização de poder político também foi evidenciada, mesmo sob a perspectiva de estabelecer relações não hierárquicas e decisões conjuntas em espaços que previam o compartilhamento de decisões entre os distintos setores, a exemplo do Grupo de Trabalho Intersetorial. Lima e VilasBôas ${ }^{26}$ analisaram o princípio da intersetorialidade por meio do estudo dos Grupos de Trabalho Municipais (GTIM) e dos Grupos de Trabalho Estaduais (GTIE) do PSE e destacaram como entraves ao processo de trabalho proposto pelo programa: o número insuficiente de profissionais que compõem os grupos municipais, a ausência de carga horária exclusiva desses grupos para a realização das ações previstas, a falta de autonomia na aplicação dos recursos financeiros e a desarticulação do planejamento municipal nos seus planos de saúde que não contemplavam um orçamento integrado.

Os limites revelados para o fortalecimento da autonomia municipal perpassavam, também, segundo outros autores, pela tenacidade em instituir padrões tradicionais de gestão que inviabilizem mudanças nos aspectos políticos, administrativos e técnicos. Whelan et al. ${ }^{27}$ demonstraram que a articulação intersetorial no processo político-gerencial para o monitoramento da implementação das ações integradas mostrou-se frágil e limitada, assim como a intersetorialidade era pouco praticada, indicando justaposição de ações entre distintas instituições governamentais.

A capacidade técnica e administrativa e a autonomia dos distintos setores envolvidos em dedicar recursos humanos, materiais e financeiros significativos para concretizar os projetos intersetoriais, direcionando as suas necessidades de acordo com valores e objetivos convergentes, instituindo uma parceria que prevê compromissos mútuos, para além de interesses conflitantes, também foram uma condição avaliada como prioritária ${ }^{28}$.

A inflexibilidade da estrutura administrativa para incorporar um novo modelo de organização, em torno de interesses articulados com outro setor e que viabilizasse, do mesmo modo, mudanças de processos administrativos foi amplamente citada. De maneira mais abrangente, um estudo sobre as práticas direcionadas à implementação de ações intersetoriais na APS comparou as rotinas organizacionais em seis municípios localizados no norte e sul da Austrália e apontou as restrições de recursos financeiros como o fator menos favorável à consecução das ações intersetoriais ${ }^{29}$.

Ao refletir sobre a necessidade de reforço da integração intersetorial, Greer e Lillvis $^{30}$ destacaram dois problemas básicos, o de coordenação e o de durabilidade. Respectivamente, as dificuldades de conseguir que as organizações relevantes incorporem novos objetivos; e, somente se os problemas de coordenação forem resolvidos, tornar esses esforços sustentáveis ao longo do tempo. A liderança política foi destacada pelo potencial de envolver um conjunto de ações dos principais decisores políticos para alterar agendas, redirecionar medidas sistêmicas, como novas políticas intersetoriais e novos procedimentos operacionais.

A necessidade de reforço das habilidades dos agentes na etapa inicial de implementação de uma política intersetorial, por meio de capacitações gradativas para viabilizar a adaptação às mudanças e a superação de modelos de políticas setorizadas e fragmentadas, foi indicada por alguns autores ${ }^{31-33}$. É importante destacar que esses estudos possuíam como fio condutor comum a discussão acerca da necessidade de fortalecimento das instituições melhorando a capacidade do pessoal para interagir com outros setores e instaurar um processo de tomada de decisões compartilhado. 
A dinâmica dos gestores diretamente envolvidos com a coordenação do processo de implementação das intervenções explicitou o desafio de compatibilizar interesses díspares em cenários de intensas disputas por recursos de poder político. As principais dificuldades relacionavam-se com a assimetria de interesses entre distintos setores, a qual pode ser considerada como o ponto mais crítico por evidenciar as intencionalidades de alguns agentes em consubstanciar práticas setoriais; e pelo fato de não encontrarem condições de possibilidades para produção de práticas articuladas, o que acaba por refletir na reprodução de modus operandi de setores específicos, possivelmente por inabilidade técnica e administrativa, tendiam a manter e desenvolver sua própria burocracia, privilegiando os meios, e não os fins (resultados).

\section{A INCIPIENTE CAPACIDADE DOS PROFISSIONAIS DE SAÚDE DA APS PARA A CONSTRUC̣ÃO DE AGENDAS INTEGRADAS E PARA A EXECUC̣ÃO DE AC̣ÕES INTEGRADAS PARA A PROMOC̣ÃO DA SAÚDE}

O principal aspecto desfavorável identificado foi a inexistência de alinhamento de agendas setoriais no início do processo de colaboração, contexto operacional e pontos de vistas setoriais dos potenciais colaboradores. Tal situação, com a expansão da ESF e sua abrangência nos diferentes territórios do País, tem revelado a fragilidade da articulação intersetorial local. Nesse sentido, a negociação de atribuições e limites de cada setor parceiro no desenvolvimento de um programa de saúde foi apontada como um dos desafios para a formalização de uma colaboração intersetorial bem-sucedida.

Nesse debate, destaca-se a compreensão da prática do trabalho em saúde ao interior da rede de serviços na APS, entendida como estruturante de um sistema hierarquizado e regionalizado, orientadora dessa rede de atenção, com integração e coordenação de um cuidado contínuo. Contudo, experiências mais recentes expuseram um cenário no qual as equipes não executavam rotineiramente ações intersetoriais e teriam também os seus esforços integrativos limitados, pois estariam na dependência da capacidade de condução da gestão local. As principais barreiras ou dificuldades diziam respeito às ações direcionadas a colocar em prática os princípios e as proposições derivadas das políticas, tendo em vista as variações vinculadas às diversas conjunturas e aos contextos onde ocorrem a implantação.

As barreiras para implementar a colaboração intersetorial foram identificadas em um estudo realizado por Trompette et al.,31 com 19 gestores municipais em dez distintos setores. Para a análise, foram consideradas a capacidade técnica, a reflexão sobre os processos de tomada de decisão e hierarquização das estruturas organizacionais e as relações de poder entre os atores sociais envolvidos. O resultado deste estudo comparativo indicou que apesar da intersetorialidade constituir uma estratégia apropriada para lidar com problemas e necessidades de saúde, ela exige estruturas organizacionais horizontais, fusão de culturas departamentais e liderança dos gestores e secretários municipais a fim de ampliar a compreensão sobre a multidimensionalidade das ações para promoção da saúde ${ }^{31}$.

Por fim, as ações intersetoriais têm sido apontadas como fundamentais para o desenvolvimento de programas para promoção da saúde, acopladas à valorização de outro elemento, a participação comunitária. A participação social é considerada um dos pilares para a promoção da saúde, cujo objetivo é a autonomia e a emancipação dos usuários, para a sua máxima inclusão social na eleição de prioridades, nas decisões e na elaboração de estratégias coerentes com as necessidades do território. Contudo, no que concerne à necessidade de envolvimento e participação comunitária na implementação de intervenções direcionadas à redução de iniquidades sociais, poucos foram os estudos voltados a esta temática-17,25-33. 
É importante destacar que esses escassos resultados demonstraram discussões relacionadas com o construto do empoderamento e integração de novas capacidades sociais para o processo de identificação de demandas, priorização de ações provenientes dos territórios, otimização da tomada de decisão e resolutividade das ações integradas. No entanto, é importante destacar que os estudos selecionados por esta revisão apresentaram a limitação de não descrever a operacionalização dessas ações nos distintos territórios adscritos à APS.

Dias et al. ${ }^{34}$ apontaram como um dos elementos associados à dificuldade de planejar e apoiar as equipes de saúde introduzir e institucionalizar uma gestão integrada, capaz de responder com eficácia à solução de problemas do território sob sua responsabilidade. Dessa forma, o dilema em operacionalizar uma política necessariamente intersetorial explicitada em um ambiente com atores que possuem agendas setoriais previamente estabelecidas, com poucos profissionais disponíveis para a correta execução das políticas, o que leva à dificuldade de conciliar os tempos institucionais dos vários setores e ao comprometimento e envolvimento setorial, limitando o alcance das ações.

Littlejohns e Smith ${ }^{35}$ indicaram a necessidade do monitoramento dos efeitos de intervenções complexas (constituída por um certo número de componentes, população, ambiente, fatores individuais e contextuais relacionados com a intervenção e com a participação comunitária). Do mesmo modo, destacaram como aspecto central a aceitação das intervenções por todos os atores sociais interessados e a disponibilidade de recursos humanos, materiais e financeiros para garantir a sustentabilidade em longo prazo.

Ainda no que se refere às condições sociopolíticas, a presença de múltiplos setores e canais de comunicação em ambos os setores envolvidos, com a evidência de distintos mecanismos operacionais das ações propostas pelas diretrizes das políticas que prevejam ações intersetoriais, foi apontada por Baum et al. ${ }^{36}$ como possível causa de alcance de diferentes resultados. Ainda que a proposição de uma intervenção intersetorial previsse um processo articulado e integrado dos agentes e setores, os estudos indicaram possíveis inconsistências e fragilidades presentes nesse percurso, tais como ausência de comprometimento igualitário entre os setores, fragmentação das ações e predomínio de abordagens setorizadas. Os autores constataram a existência de práticas multissetoriais com a presença de vários setores dedicando-se à resolução de um problema, mas sem reciprocidade, pois prevalecia a lógica e o ritmo da setorialidade que permeavam as instituições ${ }^{36}$.

A proposição de uma nova lógica, que exige um novo reordenamento das práticas profissionais e maior viabilidade política e administrativa, tem se constituído em um desafio para o âmbito local. Nessa perspectiva, as evidências corroboraram a assertiva de que as instâncias deliberativas não têm conseguido se constituir como um espaço central para fundamentar o processo de gestão de objetivos comuns, planejamento conjunto e orientação do processo de implementação das ações intersetoriais. Trata-se, por conseguinte, de um grande desafio, posto que a integração proposta pressupõe uma relação de interdependência entre agentes e instituições distintas.

Essas insuficiências foram atreladas à inexistência de um ator social com capacidade de liderança e condução suficientes para convocar todos os demais atores pertinentes a participarem da intervenção, conforme apontado por Zicker et al. ${ }^{37}$. Ademais, os mesmos autores relataram a incapacidade dos agentes envolvidos em instituir relações interinstitucionais necessárias para a execução da intervenção intersetorial e as assimetrias de capacidade decorrentes das práticas de gestão pautadas por tradições setoriais ${ }^{37}$.

Guglielmina et al. $^{\mathbf{3 8}}$, ao pesquisarem a execução de políticas intersetoriais regionais, 
apontaram que as ações ainda são incipientes e pautadas por inúmeras limitações, particularmente referentes à restrição de recursos financeiros específicos, o que, para os mencionados autores, pode conduzir ao não atendimento dos objetivos da intervenção. Todavia, os estudos analisados não abordaram evidências sistematizadas sobre o tipo de estratégias adotadas pelos decisores políticos em fases específicas e avançadas de gestão do ciclo político para possibilitar a gestão das ações articuladas. De fato, identificou-se apenas a necessidade de avaliar a factibilidade e viabilidade das agendas na articulação de mecanismos que utilizam a intersetorialidade como parâmetro para a gestão das ações integradas.

Despontaram-se, similarmente, dificuldades para a avaliação das ações e da identificação dos processos, resultados e efeitos mais completos em extensão e profundidade, sobretudo a partir dos desdobramentos das modificações de estrutura em diferentes contextos em longo prazo, as quais foram sinalizadas como uma condição praticamente inexistente (pelo menos institucionalmente) ${ }^{39}$.

O cenário supracitado contradiz os pressupostos da promoção da saúde, que expõe a necessidade de que o processo de produção do conhecimento e das políticas públicas ocorram por meio de construção e gestão compartilhadas, ou seja, por meio de ações articuladas em que haja diálogo, corresponsabilidade e cogestão entre os setores envolvidos em prol da melhoria da qualidade de vida da população. Essa problemática aponta que, para além de atentar-se às regras formais que norteiam os processos típicos das políticas públicas intersetoriais, faz-se necessário observar as relações estabelecidas e outros fatores que compõem a rotina organizacional local, que nem sempre é favorável e/ou flexível à inclusão de iniciativas para a promoção da saúde.

O conjunto de evidências expostas neste artigo demonstrou a fragilidade do âmbito local quanto à regulação da organização e aos procedimentos operacionais para implementar novas ações, sobretudo, as intersetoriais. Similarmente, indicaram que a complexidade para o fortalecimento da autonomia municipal perpassa pelo reconhecimento da potencialidade entre a articulação dos agentes de diferentes setores e institucionalidades e, também, pelo rompimento de padrões tradicionais de gestão, para promover um processo de gestão institucionalizado, orgânico e independente de mudanças dos aspectos políticos, técnicos e administrativos.

Não obstante, a sistematização permitiu identificar a formulação de diferentes estratégias para ampliar as ações e a gestão de políticas integradas em diversos contextos. A gestão de ações intersetoriais parece ser o ponto nevrálgico das experiências analisadas, pois a sua ausência explicitou o grau de dependência com outros entes federados, caso os municípios não consigam implementar mecanismos de gestão suficientemente articulados dos recursos locais.

\section{Estratégias adotadas para fomentar a implementação e gestão de ações para promoção da saúde}

Os resultados descritos em estudos empíricos recentes indicam que, na prática, as intervenções têm sido limitadas e incapazes de gerar ações integradas; mas, do mesmo modo, apontam a evolução de estratégias para a ampliação das ações para promoção da saúde em contextos diversificados. Cabe destacar algumas experiências desenvolvidas no âmbito local, que buscaram apreender fatores diferencias para o êxito das iniciativas.

A disposição de relações intergovernamentais e interorganizacionais por meio da implementação de redes regionalizadas e de novas formas de gestão das políticas públicas foi citada como uma possibilidade para o fortalecimento da autonomia ${ }^{10}$. A regionalização foi citada como uma estratégia 
capaz de permitir a agregação da capacidade dos governos locais em responder, de forma efetiva, aos problemas complexos para os quais recursos organizacionais e financeiros setorizados mostraram-se limitados.

Ante essa questão, Moretti et al. ${ }^{14}$ assinalaram a necessidade de estabelecer, em paralelo à adesão a políticas intersetoriais, protocolos que norteiem o desenvolvimento das ações articuladas entre os setores e investimentos na qualificação dos agentes, com vistas a assegurar a capacidade operacional na provisão e regulação das políticas públicas intersetoriais para a promoção da saúde no contexto municipal.

Para alguns autores, a aproximação dos profissionais envolvidos na implementação de ações intersetoriais e a discussão de estratégias para o programa proporciona um nivelamento técnico conceitual, que pode viabilizar a gestão intersetorial e a sustentabilidade da ação local 15-18. Esses estudos ressaltam, de maneira geral, que o diálogo entre os agentes de distintos setores é o passo inicial para o estabelecimento de novas relações articuladas.

A formalização de parcerias e de novos espaços para fomentar a implementação de intervenções, por meio de planos estratégicos locais que possam elencar demandas prioritárias de acordo com as necessidades sociais e de saúde de cada território de atuação, são apontadas pelos estudos como fatores favoráveis ${ }^{19}$.

O uso de indicadores comuns capazes de contribuir para a mensuração dos resultados alcançados pelas políticas integradas foram estratégias associadas à possibilidade de ampliar a identificação e análise de assimetrias administrativas e o estabelecimento de metas pactuadas. Nesse sentido, Moyses e Franco de Sá21 indicaram o método Bambu como uma estratégia para elaboração de planos locais de promoção da saúde, o qual permite a priorização e o planejamento de ações locais mediante a escuta da população e dos gestores. Os mesmos autores reforçaram que o processo de escuta dos atores e das redes sociais poderia conduzir a um processo de negociações entre os atores, setores ou instituições e ao planejamento e monitoramento contínuo de ações intersetoriais.

As proposições direcionadas à reorientação das práticas do trabalho incluíram capacitações técnicas planejadas a partir das fragilidades identificadas pela equipe, na lógica da interdisciplinaridade das ações para os profissionais da atenção e da gestão, para a melhoria da capacidade intra e interorganizacionais e para o desenvolvimento de capacidades direcionadas ao fortalecimento do planejamento integrado 22 .

Do ponto de vista da organização sociopolítica, o fortalecimento da governança local foi evidenciado como aspecto prioritário no processo de implementação da articulação entre os setores e intensificação do interesse em agir em conjunto e em prol do coletivo social, para minimizar os efeitos das mudanças organizacionais em novos ciclos políticos ${ }^{23}$.

Para a ampliação dos aspectos institucionais integrados, foi indicada a adoção da combinação de estratégias em etapas prévias ao desenvolvimento de políticas, como: a alteração dos fluxos organizacionais, novas divisões de atribuições dos atores sociais, um sistema de apoio à gestão, o estabelecimento de processos organizacionais compartilhados entre os setores ${ }^{25}$.

Outra estratégia considerada um mecanismo potente para afirmar e reforçar as relações de corresponsabilidade necessárias à produção de consensos e à tomada de decisões compartilhadas no âmbito local foi a existência de um grupo de trabalho intersetorial institucional e com funcionamento regular e contínuo ${ }^{26}$. De maneira semelhante, para outros autores, alinhar as agendas setoriais divergentes no início do processo de colaboração constitui uma condição imprescindível para o estabelecimento de intervenções articuladas ${ }^{27}$.

Como alternativa para mensurar a articulação em distintos territórios, a realização de diagnósticos situacionais, participativos e integrados às necessidades e potencialidades 
locais, que permitam a criação de espaços de trocas e negociações; e a elaboração de uma agenda de ações compartilhadas, priorizando o monitoramento e avaliação, também foram citados $^{28}$. Para tanto, os mencionados autores destacaram que o atendimento a tal prerrogativa exige a extrapolação de utilização de tecnologias médico-sanitárias e engloba tecnologias de comunicação social com o intuito de viabilizar a mobilização, organização e atuação de diferentes atores sociais na promoção e defesa de condições de vida e saúde.

O planejamento cooperativo, do mesmo modo, foi proposto como alternativa prioritária para orientar o desenvolvimento de parcerias intersetoriais, transformar os processos políticos e direcionar a construção de planos locais integrados. Um modelo interativo aplicado em diversas fases capaz de identificar os atores envolvidos, comunidade, decisores políticos do poder executivo, representantes de Organizações Não Governamentais (ONGs) e pesquisadores foi citado como uma possibilidade de avaliar os determinantes centrais das políticas. O modelo previa também a proposição de medidas para promover mudanças estruturais, como realocações intraorganizacionais de recursos (humanos, financeiros e materiais), identificação de novas fontes de financiamento, maior envolvimento dos atores vinculados aos distintos setores, bem como intensificação de contatos e cooperação entre instituições de diferentes setores ${ }^{\mathbf{2 8}}$.

Destaca-se que foi mencionada a identificação de efeitos das intervenções complexas mediante a utilização de alguns fatores de transferabilidade relacionados com a população, com o processo de implementação, com os setores envolvidos, com os contextos sociopolíticos específicos ${ }^{31}$. Contudo, os autores explicitaram que esses fatores teriam validade externa ao serem incluídos nos modelos de implementação e utilizados como ferramentas para avaliar a transferência do modelo das intervenções para outros contextos considerados semelhantes ${ }^{31}$.
Baum et al. ${ }^{36}$ assinalaram que a implementação de um processo de políticas integradas deve envolver a capacidade de negociação e de comunicação entre os distintos atores e setores, para alcançar um melhor entendimento das políticas, objetivos, linguagem, valores e prioridades dos outros setores. Os autores sugeriram que a convergência contínua de problemas e de ação estratégica pelos envolvidos oportunizam a cooperação, integração, intercâmbio de informações e o sucesso de políticas intersetoriais e, consequentemente, a permanência na agenda política e uma contínua integração entre os setores.

Uma proposta passível de potencializar as articulações intersetoriais foi discutida por Zicker et al. ${ }^{37}$, que propuseram a translação de conhecimentos por meio da aplicação destes pelos stakeholders, a fim de ampliar o alcance das ações integradas para promoção da saúde. A translação refere-se à incorporação do conhecimento produzido na implementação de práticas em um sistema de saúde como dispositivo para a melhoria de capacidades intrassetoriais e intersetoriais, por meio da otimização da gestão de dados, translação de resultados e avaliação de impactos de políticas. Para tanto, os autores propuseram a utilização do modelo de maturidade para avaliar a fase de integração entre os setores de sistemas locais por meio da identificação de características significativas, atividades explícitas, acordos de colaboração concretos e resultados visíveis, sendo possível avaliar e categorizar as fases de implementação das ações integradas, desde não reconhecida, quando não há atenção específica para o problema; até institucionalizada, quando há uma melhoria sistemática, da política e administrativa, em cada ciclo da política municipal.

Por fim, outros autores ${ }^{\mathbf{3 8}-40}$ apontaram como estratégias favoráveis para o entendimento e melhoria do processo de formulação e implementação de ações intersetoriais: (1) a presença de um planejamento local com objetivos, metas, cronograma e um prazo 
realista; (2) o compartilhamento de ações por meio de uma abordagem dinâmica e uma equipe experiente e capacitada; (3) a presença de uma liderança eficaz que direcione ações; e, (4) a disponibilidade de recursos e a tomada de decisão colaborativa.

\section{Considerações finais}

A revisão de literatura contemplou estudos produzidos no campo da saúde pública/ saúde coletiva, especialmente relacionados com as intervenções para promoção da saúde, e evidenciou os fatores relacionados com as condições contextuais restritivas para a implementação e gestão intersetorial.

A análise dos artigos selecionados permitiu assinalar duas premissas acerca da ação intersetorial para promoção da saúde. A primeira premissa é de caráter político, e propõe que a integração de diversos setores permita a busca de soluções mais integrais. Já a segunda é de natureza técnica, uma vez que acredita que a integração entre os setores permite que as diferenças entre eles possam ser usadas produtivamente para resolver problemas sociais. Ademais, a maioria dos estudos sugeriram a combinação adequada de ações que permitissem identificar os resultados atribuídos às intervenções para promoção da saúde e de políticas implementadas pelos setores relevantes a partir de diferentes domínios, gestão ou operacionalização das práticas.
Os estudos analisados, todavia, identificaram apenas a necessidade de avaliar a factibilidade e viabilidade das agendas na articulação de mecanismos que utilizam a intersetorialidade como parâmetro para a gestão das ações integradas. Portanto, o conjunto de dificuldades sinalizadas em experiências desenvolvidas para a implementação de ações e gestão integrada reforçam a necessidade de outros estudos, que possam avaliar o estabelecimento de uma intervenção concreta com ações direcionadas a promoção da saúde em territórios cobertos pela ESF.

Embora os estudos analisados por esta revisão não tenham conseguido responder a todas as questões suscitadas, remetem à necessidade de que esforços sejam somados continuamente, no intuito de superar os desafios e incertezas que se opõem a implementação e gestão de ações intersetoriais, particularmente no âmbito da APS.

\section{Colaboradores}

Prado NMBL contribuiu para elaboração do artigo com as seguintes atividades: concepção, delineamento, coleta, análise e processamento dos dados, redação da versão final do artigo. Santos AM contribuiu para elaboração do artigo com as seguintes atividades: análise e processamento dos dados, redação da versão final do artigo, contribuição significativa na revisão crítica do conteúdo e participação da aprovação da versão final do manuscrito. 


\section{Referências}

1. Lewis W. Empowerment and the ecological determinants of health: three critical capacities for practitioners. Health Promot Int [internet]. 2017 ago [acesso em 2018 maio 28]; 32(4):711-722. Disponível em: https://academic.oup.com/heapro/article $/ 32 / 4 / 711 / 2951016$.

2. Potvin L, Jones CM. Twenty-five Years After the Ottawa Charter: The Critical Role of Health Promotion for Public Health. Can J Public Health [internet]. 2011 jun [acesso em 2018 maio 28]; 102(4):244-248. Disponível em: http://journal.cpha. ca/index.php/cjph/article/view/2725.

3. Weiss D, Lillefjell M, Magnus E. Facilitators for the Development and Implementation of Health Promoting Policy and Programs: a Scoping Review at the Local Community Level. BMC Public Health. 2016 fev; 16:140.

4. Malta DC, Reis AAC, Jaime PC, et al. O SUS e a Política Nacional de Promoção da Saúde: perspectiva resultados, avanços e desafios em tempos de crise. Ciênc Saúde Colet., Rio de Janeiro, v. 23, n. 6, p.17991809, jun. 2018. Disponível em: http://dx.doi. org/10.1590/1413-81232018236.04782018.

5. Giovanella L, Mendonça MHM, Almeida PF, et al. Saúde da Família: limites e possibilidades para uma abordagem integral à saúde no Brasil. Ciênc Saúde Colet. 2009; 14(3):783-794.

6. Albuquerque TIP, Franco de Sá RMP, Araújo JLAC. Perspectivas e desafios da nova Política Nacional de Promoção da Saúde: para qual arena política aponta a gestão. Ciênc Saúde Colet. [internet]. 2016 jun [acesso em 2018 maio 28]; 21(6):1695-1170. Disponível em: http://dx.doi.org/10.1590/141381232015216.07672016 .

7. Teixeira MB, Casanova A, Oliveira CCM, et al. Avaliação das práticas de promoção da saúde: um olhar das equipes participantes do Programa Nacional de Melhoria do Acesso e da Qualidade da Atenção Básica. Saúde debate. 2014 out; 38(esp):52-68.
8. Heidemann ITSB, Cypriano CC, Gastaldo D, et al. Estudo comparativo de práticas de promoção da saúde na atenção primária em Florianópolis, Santa Catarina, Brasil e Toronto, Ontário, Canadá. Cad. Saúde Pública [internet]. 2018 [acesso em 2018 jan 28]; 34(4):1-13. Disponível em: http://www.scielo.br/scielo.php?script=sci_arttext $\&$ pid $=$ S0102$-311 X 2018000405013 \& \operatorname{lng}=$ pt.

9. Storm I, Harting J, Stronks K, et al. Measuring stages of health in all policies on a local level: The applicability of a maturity model. Health Policy. 2014; 114(2-3):183-191.

10. Peckham S, Hann A, Kendall S, et al. Health promotion and disease prevention in general practice and primary care: A scoping study. Prim Health Care Res Dev. 2017; 18(6):529-540.

11. Tong A, Sainsbury P, Craig J. Consolidated criteria for reporting qualitative research (COREQ): a 32item checklist for interviews and focus groups. Int J Qual Health Care. 2007; 19(6):349-357.

12. Giovanella L, Mendonça MHM, Almeida PF, et al. Saúde da família: limites e possibilidades para uma abordagem integral de atenção primária à saúde no Brasil. Ciênc Saúde Colet. [internet]. 2009 [acesso em 2018 maio 28]; 14(3):783-794. Disponível em: http://dx.doi.org/10.1590/S141381232009000300014 .

13. Horta NC, Sena RR, Silva Maria EO, et al. A prática das equipes de saúde da família: desafios para a promoção de saúde. Rev Bras Enferm [internet]. 2009 [acesso em 2018 jan 25]; 62(4):524-529. Disponível em: http://dx.doi.org/10.1590/S003471672009000400005 .

14. Moretti AC, Teixeira FF, Suss FMB, et al. Intersetorialidade nas ações de promoção de saúde realizadas pelas equipes de saúde bucal de Curitiba (PR). Ciênc Saúde Colet. [internet]. 2010 [acesso em 2018 jan 12]; 15(Supl.1):1827-1834. Disponível em: http:// dx.doi.org/10.1590/S1413-81232010000700095. 
15. Magalhães R, Bodstein R. Avaliação de iniciativas e programas intersetoriais em saúde: desafios e aprendizados. Ciênc Saúde Colet. [internet]. 2009 [acesso em 2018 jan 22]; 14(3):861-868. Disponível em: http://dx.doi.org/10.1590/S141381232009000300021 .

16. Silva KS, Rodrigues AT. Ações intersetoriais para promoção da saúde na Estratégia Saúde da Família: experiências, desafios e possibilidades. Rev Bras Enferm [internet]. 2010 [acesso em 2018 jan 20]; 63(5):762-769. Disponível em: http://www.scielo.br/scielo.php?script=sci_arttext $\&$ pid $=$ S0034$-71672010000500011 \& \operatorname{lng}=\mathrm{en}$.

17. Freitas MLA, Mandú ENT. Promoção da saúde na Estratégia Saúde da Família: análise de políticas de saúde brasileiras. Acta Paul Enferm [internet]. 2010 abr [acesso em 2017 dez 10]; 23(2):200-205. Disponível em: http://dx.doi.org/10.1590/S010321002010000200008 .

18. Medina MG, Aquino R, Vilasbôas ALQ, et al. Promoção da saúde e prevenção de doenças crônicas: o que fazem as equipes de Saúde da Família. Saúde debate [internet]. 2014 [acesso em $2017 \mathrm{dez}$ 10]; 38(esp):6982. Disponível em: http://dx.doi.org/10.5935/0103-1104.2014 S006.

19. Littlejohns L, Smith BN. Building bridges between health promotion and social sustainability: an analysis of municipal policies in Western Canada. Local Environment. 2013; 19(4):449-468.

20. Heidemann ITSB, Wosny AM, Boehs AE. Promoção da Saúde na Atenção Básica: estudo baseado no método de Paulo Freire. Ciênc Saúde Colet. [internet].2014 [acesso em 2017 dez 10]; 19(8):3553-3559. Disponível em: http://dx.doi.org/10.1590/141381232014198.11342013

21. Moysés ST, Franco de Sá R. Planos locais de promoção da saúde: intersetorialidade(s) construída(s) no território. Ciênc Saúde Colet. [internet]. 2014 [acesso em 2017 dez 10]; 19(11):4323-4330. Disponível em: http://dx.doi.org/10.1590/1413812320141911.11102014.
22. Cavalcanti AD, Cordeiro JC. As ações intersetoriais na Estratégia de Saúde da Família: um estudo da representação do conceito de saúde e de suas práticas na Atenção Básica. Rev Bras Med Família Comun. 2015; 10(37):1-9.

23. Silva DAJ, Tavares MFL. Ação intersetorial: potencialidades e dificuldades do trabalho em equipes da Estratégia Saúde da Família na cidade do Rio de Janeiro. Saúde debate [internet]. 2016 [acesso em 2017 dez 10]; 40(111):193-205. Disponível em: http://www.scielo.br/scielo.php?script=sci arttext\&pid=S0103-11042016000400193\&lng=en .

24. Rodrigues CC, Ribeiro KSQS. Promoção da saúde: a concepção dos profissionais de uma unidade de saúde da família. Trab Educ Saúde [internet]. 2012 [acesso em 2018 jan 10]; 10(2):235-255. Disponível em: https://dx.doi.org/10.1590/S198177462012000200004 .

25. Azevedo E, Pelicioni MCF, Westphal MF. Práticas intersetoriais nas políticas públicas de promoção de saúde. Physis [internet]. 2012 [acesso em $2017 \mathrm{dez}$ 10]; 22(4):1333-1356. Disponível em: http://www. scielo.br/scielo.php?script=sci_arttext\&pid=S0103$-73312012000400005 \& \operatorname{lng}=$ en.

26. Lima EC, Vilasbôas ALQ. Implantação das ações intersetoriais de mobilização social para o controle da dengue na Bahia, Brasil. Cad. Saúde Pública [internet]. 2011 [acesso em 2018 maio 28]; 27(8):1507-1519. Disponível em: http://www.scielo.br/scielo.php?script=sci_arttext\&pid=S0102 $-311 X 2011000800006 \& \operatorname{lng}=$ en.

27. Whelan J, Love P, Pettman T, et al. Cochrane Update: Predicting sustainability of intervention effects in public health evidence: identifying key elements to provide guidance. J Public Health. 2015 jun; 36(2):347-351

28. Rütten A, Gelius P. Building policy capacities: an interactive approach for linking knowledge to action in health promotion. Health Promotion Internet 2014 set; 29(3):569-582. 
29. Anaf J, Baum F, Freeman T, et al. Factors shaping intersectoral action in primary health care services. Aust N Z J Public Health. 2014; 38(6):553-559.

30. Greer SL, Lillvis DF. Beyond leadership: political strategies for coordination in health policies. Health Policy [internet]. 2014 maio [acesso em 2018 jan 14]; 116(1):12-17. Disponível em: https://www.ncbi. nlm.nih.gov/pubmed/24576497.

31. Trompette J, Kivits J, Minary L, et al. Stakeholders' perceptions of transferability criteria for health promotion interventions: a case study. BMC Public Health. 2014; 14:1134-1140.

32. Tess BH, Aith FMA. Intersectorial health-related policies: the use of a legal and theoretical framework to propose a typology to a case study in a Brazilian municipality. Ciênc Saúde Colet. 2014 nov; 19(11):4449-4456.

33. Hendriks A, Jansen MWJ, Gubbels JS, et al. Local government officials views on intersectoral collaboration within their organization - A qualitative exploration. Health Policy Technol. 2015 mar; 4(1):47-57.

34. Dias MSA, Parente JRF, Vasconcelos MIO, et al. Intersetorialidade e Estratégia Saúde da Família: tudo ou quase nada a ver. Ciênc Saúde Colet [internet]. 2014 nov [acesso em $2017 \mathrm{dez}$ 10]; 19(11):4371-4382. Disponível em: http://dx.doi.org/10.1590/1413812320141911.11442014.
35. Littlejohns LB, Smith N. Building bridges between health promotion and social sustainability: an analysis of municipal policies in Western Canada. Intern J Justice Sustainability. 2014; 19(4):449-468.

36. Baum F, Angela L, Toni D, et al. Evaluation of Health in All Policies: concept, theory and application. Health Promotion Inter. 2014; 29(supl.1):130-142.

37. Zicker F, Faid M, Reeder J, et al. Building coherence and synergy among global health initiatives. Health Res Policy Syst. 2015; 13:75-82.

38. Weiss D, Lillefjell M, Magnus E. Facilitators for the development and implementation of health promoting policy and programs - a scoping review at the local community level. BMC Public Health.2016; 16:140-151.

39. Kothari A, McPherson C, Gore D, et al. A multiple case study of intersectoral public health networks: experiences and benefits of using research. Health Res Policy Syst. 2016; 14:11-22.

40. Guglielmin M, Muntaner C, O'Campo P, et al. A scoping review of the implementation of health in all policies at the local level. Health Policy. 2018; 122:284-292.

Recebido em: 30/05/2018

Aprovado em: 23/08/2018

Conflito de interesses: inexistente

Suporte financeiro: não houve 